\title{
On a Generalization of the Midpoint Rule*
}

\section{By Franz Stetter}

I. Introduction. A modified midpoint rule for the approximate calculation of weighted integrals $\int_{a}^{b} p(x) f(x) d x$, where $p(x) \geqq 0$ is the weight function, has been recently proposed by Jagermann [1]. Although this formula reduces to the common midpoint rule in the particular case $p(x) \equiv 1$, in the general case of arbitrary weight functions the error does not vanish for all polynomials $\alpha+\beta . x$. The purpose of this paper is to generalize the midpoint rule such that the formula is exact for polynomials of first degree and arbitrary weight function $p(x) \geqq 0$.

In view of practical calculations, the repeated midpoint rule is very useful because of its simplicity and small round-off error. Moreover, an error estimation does not require higher derivatives whose bounds are often not easy to obtain. For a comparison of the repeated midpoint rule to both Gaussian quadratures and "best" quadratures we refer to Stroud and Secrest [2].

II. Generalized Midpoint Rule. We assume that the weight function $p(x)$ does not identically vanish on any subinterval of $[a, b]$. Let

$$
y=H(x)=\int_{a}^{x} p(t) d t, \quad H(b)=1,
$$

and let the inverse function of $H$ (which exists because $H(x)$ is monotonic increasing) be denoted by $L$ :

$$
x=L(y)=H^{-1}(y) .
$$

For $i=0,1, \cdots, N-1,(N \geqq 1)$ we put

$$
a_{i}=N \int_{i / N}^{(i+1) / N} L(y) d y=N \int_{x_{i}}^{x_{i+1}} t p(t) d t
$$

where $x_{i}=L(i / N)$. We now define the generalized rule by:

$$
\int_{a}^{b} p(x) f(x) d x=\frac{1}{N} \sum_{i=0}^{N-1} f\left(a_{i}\right)+R_{N} .
$$

Assuming $f \in C^{2}[a, b]$ the error $R_{N}$ can be expressed by

$$
R_{N}=\frac{1}{2}\left(\int_{a}^{b} x^{2} p(x) d x-\frac{1}{N} \sum_{i=0}^{N-1} a_{i}{ }^{2}\right) f^{\prime \prime}(\xi)=\frac{1}{2} C_{N} f^{\prime \prime}(\xi), \quad a<\xi<b .
$$

I'roof. Dividing $[a, b]$ into the subintervals $\left[x_{i}, x_{i+1}\right]$ we obtain for the error $R_{N}$

$$
R_{N}=\sum_{i=0}^{N-1}\left\{\int_{x_{i}}^{x_{i+1}} p(x) f(x) d x-\frac{1}{N} f\left(a_{i}\right)\right\} .
$$

Received October 30, 1967.

* Work performed under the auspices of the United States Atomic Energy Commission. 
By the Taylor series

$$
f(x)=f\left(a_{i}\right)+\left(x-a_{i}\right) f^{\prime}\left(a_{i}\right)+\frac{1}{2}\left(x-a_{i}\right)^{2} f^{\prime \prime}\left(\xi_{i}\right)
$$

and by (3) we get the expression

$$
\begin{aligned}
R_{N} & =\frac{1}{2} \sum_{i=0}^{N-1}\left\{\int_{x_{i}}^{x_{i+1}}\left(x-a_{i}\right)^{2} p(x) f^{\prime \prime}\left(\xi_{i}\right) d x\right\} \\
& =\frac{1}{2}\left(\sum_{i=0}^{N-1} \int_{x_{i}}^{x_{i+1}}\left(x-a_{i}\right)^{2} p(x) d x\right) f^{\prime \prime}(\xi) .
\end{aligned}
$$

Furthermore, it follows from (3) that

$$
\begin{aligned}
\sum_{i=0}^{N-1} \int_{x_{i}}^{x_{i+1}}\left(x-a_{i}\right)^{2} p(x) d x & =\sum_{i=0}^{N-1}\left\{\int_{x_{i}}^{x_{i+1}} x^{2} p(x) d x-\frac{2}{N} a_{i}{ }^{2}+\frac{1}{N} a_{i}{ }^{2}\right\} \\
& =\int_{a}^{b} x^{2} p(x) d x-\frac{1}{N} \sum_{i=0}^{N-1} a_{i}{ }^{2}
\end{aligned}
$$

(6) and (7) yield the bound (5).

$C_{N}$ can also be interpreted as the integration error of the function $f=x^{2}$. It may be noted that Jagermann's modification of the midpoint rule is obtained if the integral $N \int_{i / N}^{(i+1) / N} L(y) d y$ in (3) is approximated by the (ordinary) midpoint rule, i.e., by $L((2 i+1) / 2 N)$.

\section{Examples.}

(a) For $p(x) \equiv 1$ and $a=0, b=1$, we obtain $a_{i}=(2 i+1) / 2 N$ and, from (5), $C_{N}=1 / 12 N^{2}$ in accordance with the common midpoint rule.

(b) Let $p(x)=\pi^{-1}\left(1-x^{2}\right)^{-1 / 2}$ and $a=-1, b=1$. From $L(y)=-\cos \pi y$ it immediately follows that:

$$
a_{i}=-\frac{2 N}{\pi} \sin \frac{\pi}{2 N} \cos \frac{2 i+1}{2 N} \pi \quad(i=0, \cdots, N-1)
$$

and

$$
\begin{aligned}
C_{N}=\frac{1}{2}-\frac{1}{N} \sum_{i=0}^{N-1} a_{i}^{2} & =\frac{1}{2} \quad \text { for } N=1 \\
& =\frac{1}{2}-\frac{2 N^{2}}{\pi^{2}} \sin ^{2} \frac{\pi}{2 N} \quad \text { for } N \geqq 2 .
\end{aligned}
$$

Obviously, $C_{N}=O\left(N^{-2}\right)$.

(c) For the infinite interval $a=0, b=\infty$ and the weight function $p(x)=e^{-x}$ we get from $L(y)=-\log (1-y)$ :

$$
\begin{gathered}
a_{N-1}=1+\log N \\
a_{i}=1+\log N-(N-i-1) \log (N-i-1)-(N-i) \log (N-i)
\end{gathered}
$$

for $i=0,1, \cdots, N-2$. Numerically computed values of $C_{N}$ 
ON A GENERALIzATION OF THE MIDPOINT RULE

\begin{tabular}{|c|c|c|c|c|c|c|}
\hline$N$ & 1 & 2 & 5 & 10 & 20 & 50 \\
\hline$C_{N}$ & 1.000 & 0.520 & 0.213 & 0.108 & 0.054 & 0.022 \\
\hline
\end{tabular}

show that $C_{N}$ goes to 0 with the order $O\left(N^{-1}\right)$.

Applied Mathematics Division

Argonne National Laboratory

Argonne, Illinois 60439

1. D. JAGermanN, "Investigation of a modified mid-point quadrature formula," Math. Comp., v. 20, 1966, pp. 79-89. MR $32 \# 8499$.

2. A. H. Stroud \& D. SeCrest, Gaussian Quadrature Formulas, Prentice-Hall, Englewood Cliffs, N. J., 1966. MR 34 \#2185. 